\title{
On Using Exponential Parameter Estimators with an Adaptive Controller
}

\author{
Parag Patre and Suresh M. Joshi
}

\begin{abstract}
Typical adaptive controllers are restricted to using a specific update law to generate parameter estimates. This paper investigates the possibility of using any exponential parameter estimator with an adaptive controller such that the system tracks a desired trajectory. The goal is to provide flexibility in choosing any update law suitable for a given application. The development relies on a previously developed concept of controller/update law modularity in the adaptive control literature, and the use of a converse Lyapunov-like theorem. Stability analysis is presented to derive gain conditions under which this is possible, and inferences are made about the tracking error performance. The development is based on a class of Euler-Lagrange systems that are used to model various engineering systems including space robots and manipulators.
\end{abstract}

\section{INTRODUCTION}

Control laws with adaptive feedforward terms have been widely used to compensate for the linear-in-the-parameter (LP) uncertainty in nonlinear systems. The update law or a parameter estimator is an integral part of an adaptive controller that generates parameter estimates used by the adaptive controller. The convergence properties of the estimator directly impact the tracking error performance, even though asymptotic tracking may still be possible without the estimates converging to their true values. Lyapunovbased techniques are commonly used to design and analyze an adaptive control law and its corresponding parameter update law, where the controller and the update law are designed in conjunction with one another such that the update law cancels certain cross terms in the stability analysis to make the overall time-derivative of the Lyapunov function negative. This restrains the design of the update law to one specific form, which typically is a gradient update law. Gradient update laws are notorious for exhibiting slower parameter convergence [1]-[3], which could lead to a slower transient performance of the tracking error in comparison to other possible adaptive update laws (e.g., least-squares or recursive least-squares update law [1], [3]-[5]). In order to overcome this issue of slow convergence, several results have been developed in the literature that aim to modify or augment the typical tracking error-based gradient update law. For example, composite adaptive update laws [1], [2], [6];

This research was supported in part by an appointment to the NASA Postdoctoral Program at Langley Research Center, administered by Oak Ridge Associated Universities through a contract with NASA.

Parag Patre is NASA Postdoctoral Program (NPP) Fellow at NASA Langley Research Center, Hampton, VA 23681, USA parag.patrelnasa.gov

Suresh M. Joshi is Senior Scientist for Control Theory at NASA Langley Research Center, Hampton, VA 23681, USA suresh.m.joshi@nasa.gov prediction error (a predicted value of the difference between the actual parameter and its estimate)-based update laws [7][11]; and various least-squares update laws [3], [5], [12]. In addition, several results have discussed the robustness and exponential convergence properties of estimation schemes [1], [4], [5], [13], [14]. However, the use of these update laws is still contingent upon their ability to cancel cross terms in the Lyapunov-based stability analysis. That is, only a specific type of update law can be used with the adaptive controller. This raises the question if we can have flexibility in choosing a parameter update law best suited for a given application to potentially achieve a better performance.

Intuitively, if a parameter estimator has an exponential convergence, it could be combined with the adaptive controller to achieve tracking. However, as mentioned earlier, stability cannot be guaranteed unless it is designed to cancel certain cross terms. The result in this paper is motivated by the desire to investigate if a formal proof can be presented for guaranteeing stability and tracking performance if any such exponential parameter estimator is used with the adaptive controller. In the existing literature, there are various results on a class of modular adaptive controllers that provide such controller/update law separation (cf. [7], [9]-[11]). One approach is to use nonlinear damping [8], [15] to yield a strong controller that is input-to-state stable (ISS) with respect to the parameter estimation error. This enables the use of any update law that yields bounded parameter estimates. A brief survey of nonlinear damping-based modular adaptive control results is provided in [7]. Another approach was investigated in [16] by designing a robust control law using the RISE (robust integral of the sign of the error [17]) feedback method, such that a generic form of an update law in terms of tracking errors could be used.

In this paper, modularity in the update law (i.e., controller/identifier separation), and tracking is achieved in three steps. First, for a system which is linear in uncertain constant parameters, an adaptive controller is developed and augmented with a nonlinear damping term. This makes the system ISS with respect to the estimation error. That is, for a bounded parameter estimation error, the tracking error is bounded. Second, a converse Lyapunov-like theorem is presented, and existence of a Lyapunov-like function is shown for systems with trajectories that are exponentially decaying to a small bound. Third, any parameter estimator that gives a parameter estimation error exponentially decaying to a small bound is used with the adaptive controller. Based on the second step, the existence of a Lyapunov-like function for such a parameter estimator is guaranteed. This combined 
with the first step is used to prove a tracking result under some sufficient gain conditions such that, if the parameter estimation error is uniformly ultimately bounded (UUB), then the tracking error is also UUB.

Typical adaptive control designs yield asymptotic tracking without any tracking performance guarantees. In contrast, the current result proves only UUB tracking, but guarantees performance bounds on the tracking error while allowing the use of a broad class of exponential estimators. Moreover, if the parameter estimator has an exponential convergence to zero, the tracking errors also converge to zero exponentially. Compared to the previous results on controller/update law modularity, the current result can be considered as an alternative choice by the control design engineers. The result in this paper achieves modularity in the controller/update law using ISS arguments similar to [7]-[11] by including a nonlinear damping term in the torque control input. However, in [7][11], in order to prove tracking, additional assumptions about the update law itself had to be made. For example, it needed to be driven by a prediction error, which must be square integrable; the update law must be bounded, etc. This in turn required extra terms in the control law. Moreover, the estimator must also ensure that the estimate of the inertia matrix be positive definite. In most cases, this required the use of projection algorithms, thus leading to an increased complexity, and even though asymptotic tracking was proved, no bounds on the tracking error transient could be derived. In contrast, the result in this paper only assumes that the parameter identification scheme gives an estimation error that exponentially decreases to a small bound, and no additional assumptions are required.

Since the objective is to design an adaptive control law that utilizes parameter estimates generated by any estimator with exponential convergence, design of a specific estimator is not considered in this work. It should be noted that the construction of any such practical estimator may require additional inputs, such as bounds on measurement noise, bound on estimation error, etc. [1], [4], [5], [13], [14].

\section{Dynamic Model And Properties}

The class of nonlinear dynamic systems considered in this paper can be described by the following Euler-Lagrange formulation, which can be used to model a large class of engineering systems such as space robots and manipulators [18], [19]:

$$
M(q) \ddot{q}+V_{m}(q, \dot{q}) \dot{q}+G(q)+F(\dot{q})=\tau(t) .
$$

In (1), $M(q) \in \mathbb{R}^{n \times n}$ denotes the inertia matrix, $V_{m}(q, \dot{q}) \in$ $\mathbb{R}^{n \times n}$ denotes the centripetal-Coriolis matrix, $G(q) \in \mathbb{R}^{n}$ denotes the gravity vector, $F(\dot{q}) \in \mathbb{R}^{n}$ denotes friction, $\tau(t) \in \mathbb{R}^{n}$ represents the torque input control vector, and $q(t), \dot{q}(t), \ddot{q}(t) \in \mathbb{R}^{n}$ denote the generalized position, velocity, and acceleration vectors, respectively. The subsequent development is based on the assumption that $q(t)$ and $\dot{q}(t)$ are measurable and that $M(q), V_{m}(q, \dot{q}), G(q)$, and $F(\dot{q})$ are unknown. Throughout the paper $|\cdot|$ denotes the absolute value of the scalar argument, $\|\cdot\|$ denotes the standard Euclidean norm for a vector or the induced norm for a matrix. The following properties will be exploited in the subsequent development.

Property 1: The inertia matrix $M(q)$ is symmetric, positive definite. Therefore, it satisfies the following inequality $\forall q(t), \xi(t) \in \mathbb{R}^{n}[18],[19]:$

$$
m_{1}\|\xi\|^{2} \leq \xi^{T} M(q) \xi \leq m_{2}\|\xi\|^{2}
$$

where $m_{1}, m_{2} \in \mathbb{R}$ are known positive constants.

Property 2: The time derivative of inertia matrix $\dot{M}(q)$ and the centripetal-Corilois matrix $V_{m}(q, \dot{q})$ satisfy the following skew-symmetry property $\forall q(t), \dot{q}(t), \xi(t) \in \mathbb{R}^{n}$ [18], [19]:

$$
\xi^{T}\left(\frac{1}{2} \dot{M}(q)-V_{m}(q, \dot{q})\right) \xi=0 .
$$

\section{Control Objective And Development}

The objective is to design an adaptive controller with the flexibility of using any parameter estimator that has an exponential parameter convergence to a small error bound, such that the system tracks a desired time-varying trajectory $q_{d}(t)$ despite uncertainties in the dynamic model. To quantify this objective, a position tracking error, denoted by $e_{1}(t) \in$ $\mathbb{R}^{n}$, is defined as

$$
e_{1} \triangleq q_{d}-q
$$

In addition, to facilitate the subsequent analysis, a filtered tracking error [18], $e_{2}(t) \in \mathbb{R}^{n}$, is defined as

$$
e_{2} \triangleq \dot{e}_{1}+\alpha_{1} e_{1}
$$

where $\alpha_{1} \in \mathbb{R}$ denotes a positive constant. Differentiating (5) with respect to time, and premultiplying the resulting expression with $M(q)$ yields

$$
M(q) \dot{e}_{2}=M(q) \ddot{q}_{d}-M(q) \ddot{q}+M(q) \alpha_{1} \dot{e}_{1} .
$$

Using (1), (4), (5) in (6), we get

$$
M(q) \dot{e}_{2}=Y \theta-\tau(t)-V_{m}(q, \dot{q}) e_{2}
$$

where the following property is used:

Property 3: A part of the dynamics in (1) can be linearly parameterized as [18], [20]

$$
\begin{aligned}
Y \theta= & M(q) \ddot{q}_{d}+V_{m}(q, \dot{q})\left(\dot{q}_{d}+\alpha_{1} e_{1}\right)+G(q) \\
& +F(\dot{q})+M(q) \alpha_{1} \dot{e}_{1}
\end{aligned}
$$

where $\theta \in \mathbb{R}^{p}$ contains the constant unknown system parameters (e.g., component masses, moments of inertia, damping and friction coefficients, etc.), and $Y\left(q, \dot{q}, \dot{q}_{d}, \ddot{q}_{d}\right) \in \mathbb{R}^{n \times p}$ is a regression matrix that contains known nonlinear functions of the known generalized coordinates $q(t), \dot{q}(t)$ and desired trajectories.

Based on the open-loop error system in (7), the torque control input is designed as

$$
\tau(t)=Y \hat{\theta}(t)+k_{1} e_{2}(t)+k_{n}\|Y\|^{2} e_{2}(t)
$$

where $k_{1}, k_{n} \in \mathbb{R}$ are positive constants, $\hat{\theta}(t) \in \mathbb{R}^{p}$ is an adaptive estimate of $\theta$. Nonlinear damping terms, similar to $k_{n}\|Y\|^{2} e_{2}(t)$ in the control input, have been previously 
used in results such as [8], [10]. Substituting (9) in to (7), we get the following closed-loop error system:

$$
M(q) \dot{e}_{2}=Y \tilde{\theta}-k_{1} e_{2}-k_{n}\|Y\|^{2} e_{2}-V_{m}(q, \dot{q}) e_{2}
$$

where $\tilde{\theta}(t) \in \mathbb{R}^{p}$ is the parameter estimation error defined as

$$
\tilde{\theta}(t)=\theta-\hat{\theta}(t) .
$$

\section{INPUT-TO-STATE STABILITY}

In this section, we show the ISS property of the system with respect to the parameter estimation error $\tilde{\theta}(t)$. This allows controller/update law modularity, and thus, any update law that generates bounded parameter estimates can be used with the adaptive controller. We now have the theorem:

Theorem 1: The closed-loop error system in (10) is inputto-state stable with respect to $\tilde{\theta}(t)$, i.e., if $\|\tilde{\theta}(t)\| \in \mathcal{L}_{\infty}$, then $\left\|e_{1}(t)\right\| \in \mathcal{L}_{\infty}$.

Proof: Define a Lyapunov function candidate

$$
V_{\text {iss }}=\frac{1}{2} e_{2}^{T} M(q) e_{2} .
$$

Using (2), $V_{i s s}(t)$ can be lower and upper bounded as

$$
\frac{1}{2} m_{1}\left\|e_{2}\right\|^{2} \leq V_{i s s} \leq \frac{1}{2} m_{2}\left\|e_{2}\right\|^{2} .
$$

Taking the time derivative of (11), and substituting from (10), we get

$$
\begin{aligned}
\dot{V}_{i s s}= & e_{2}^{T}\left[Y \tilde{\theta}-k_{1} e_{2}-k_{n}\|Y\|^{2} e_{2}-V_{m}(q, \dot{q}) e_{2}\right](13) \\
& +\frac{1}{2} e_{2}^{T} \dot{M}(q) e_{2} .
\end{aligned}
$$

Using the skew-symmetry property (3), the expression in (13) can be upper bounded as

$$
\dot{V}_{i s s} \leq-k_{1}\left\|e_{2}\right\|^{2}+\left\|e_{2}\right\|\|Y\|\|\tilde{\theta}\|-k_{n}\left\|e_{2}\right\|^{2}\|Y\|^{2} .
$$

Completing the squares for the last two terms

$$
\dot{V}_{i s s} \leq-k_{1}\left\|e_{2}\right\|^{2}-k_{n}\left(\|Y\|\left\|e_{2}\right\|-\frac{1}{2 k_{n}}\|\tilde{\theta}\|\right)^{2}+\frac{1}{4 k_{n}}\|\tilde{\theta}\|^{2}
$$

therefore,

$$
\dot{V}_{i s s} \leq-k_{1}\left\|e_{2}\right\|^{2}+\frac{1}{4 k_{n}}\|\tilde{\theta}\|^{2} .
$$

Using (12) in (14), the upper bound on $\dot{V}_{i s s}(t)$ can be expressed as

$$
\dot{V}_{i s s} \leq-2 \lambda_{i s s} V_{i s s}+\frac{1}{4 k_{n}}\|\tilde{\theta}\|^{2}
$$

where

$$
\lambda_{i s s}=\frac{k_{1}}{m_{2}} .
$$

After solving the differential inequality in (15), we get

$$
V_{i s s}(t) \leq V_{i s s}(0) e^{\left(-2 \lambda_{i s s} t\right)}+\frac{1}{8 \lambda_{i s s} k_{n}} \sup _{0 \leq \tau \leq t}\|\tilde{\theta}(\tau)\|^{2} .
$$

Hence, from (12) and (16)

$$
\begin{aligned}
\left\|e_{2}(t)\right\|^{2} \leq & \frac{m_{2}}{m_{1}}\left\|e_{2}(0)\right\|^{2} e^{\left(-2 \lambda_{i s s} t\right)} \\
& +\frac{1}{4 m_{1} \lambda_{i s s} k_{n}} \sup _{0 \leq \tau \leq t}\|\tilde{\theta}(\tau)\|^{2} .
\end{aligned}
$$

Using the fact that $a^{2} \leq b^{2}+c^{2} \Rightarrow a \leq b+c$ for all positive scalars $a, b, c,(17)$ can be expressed as

$$
\begin{aligned}
\left\|e_{2}(t)\right\| \leq & \sqrt{\frac{m_{2}}{m_{1}}}\left\|e_{2}(0)\right\| e^{\left(-\lambda_{i s s} t\right)} \\
& +\frac{1}{2 \sqrt{m_{1} \lambda_{i s s} k_{n}}} \sup _{0 \leq \tau \leq t}\|\tilde{\theta}(\tau)\| .
\end{aligned}
$$

The bound in (18) indicates input-to-state stability (ISS) (cf. Def 4.7, pp. 175 [21]). That is, if $\sup _{0 \leq \tau \leq t}\|\tilde{\theta}(\tau)\|$ is bounded, then $\left\|e_{2}(t)\right\|$ is bounded, and therefore, from (5), the tracking error $\left\|e_{1}(t)\right\|$ is bounded.

Any update law that guarantees a bounded $\|\tilde{\theta}\|$ can be used. Further, if $\|\tilde{\theta}(t)\|$ is UUB, then subsequently it will be shown (Theorem 3) that the tracking error is UUB. First we prove the following converse Lyapunov-like theorem.

\section{Converse Lyapunov-LiKe Theorem}

The following can be proved directly by extending the result in Theorem 4.14 of [21].

Theorem 2: Consider a nonlinear system

$$
\dot{x}(t)=f(t, x)
$$

where $f:[0, \infty) \times D \rightarrow \mathbb{R}^{n}$ is continuously differentiable, $D=\left\{x \in \mathbb{R}^{n} \mid\|x\|<r\right\}$ for some $r$, and the Jacobian matrix $[\partial f / \partial x]$ is uniformly bounded on $D$. Let $x=0$ be an equilibrium point for the system in (19). Let $k, \lambda, \varepsilon$, and $r_{0}$ be positive constants with $r_{0}<r / k$. Let $D_{0}=\{x \in$ $\left.\mathbb{R}^{n} \mid\|x\|<r_{0}\right\}$. Assume that $\forall x\left(t_{0}\right) \in D_{0}, \forall t \geq t_{0} \geq 0$, the trajectories of the system in (19) satisfy

$$
\|x(t)\| \leq k\left\|x\left(t_{0}\right)\right\| e^{-\lambda\left(t-t_{0}\right)}+\varepsilon .
$$

Then, there exists a function $V:[0, \infty) \times D_{0} \rightarrow \mathbb{R}$ that satisfies the inequalities

$$
\begin{gathered}
c_{1}\|x\|^{2} \leq V(t, x) \leq c_{2}\|x\|^{2}+c_{3}\|x\|+c_{4} \\
\frac{\partial V}{\partial t}+\frac{\partial V}{\partial x} f(t, x) \leq-c_{5}\|x\|^{2}+c_{6}\|x\|+c_{7}
\end{gathered}
$$

for some positive constants $c_{1}, c_{2}, \ldots, c_{7}$. Moreover, if $r=$ $\infty$ and the origin is globally uniformly ultimately bounded, then $V(t, x)$ is defined and satisfies the aforementioned inequalities on $\mathbb{R}^{n}$. Furthermore, if the system is autonomous, $V$ can be chosen independent of $t$.

Proof: Let $\phi(\tau ; t, x)$ denote the solution of the system starting at $(t, x)$; i.e., $\phi(t ; t, x)=x$. For all $x \in D_{0}, \phi(\tau ; t, x) \in$ $D$ for all $\tau \geq t$. Let

$$
V(t, x)=\int_{t}^{t+\delta} \phi^{T}(\tau ; t, x) \phi(\tau ; t, x) d \tau
$$


where $\delta$ is a positive constant to be chosen. Due to exponentially decaying bound on the trajectories, we have

$$
\begin{aligned}
V(t, x)= & \int_{t}^{t+\delta}\|\phi(\tau ; t, x)\|^{2} d \tau \\
\leq & \int_{t}^{t+\delta}\left(k^{2}\|\phi(t ; t, x)\|^{2} e^{-2 \lambda(\tau-t)}\right. \\
& \left.\quad+2 \varepsilon k\|\phi(t ; t, x)\| e^{-\lambda(\tau-t)}+\varepsilon^{2}\right) d \tau
\end{aligned}
$$

Using the fact $\phi(t ; t, x)=x$,

$V(t, x) \leq \frac{k^{2}}{2 \lambda}\left(1-e^{-2 \lambda \delta}\right)\|x\|^{2}+\frac{2 \varepsilon k}{\lambda}\left(1-e^{-\lambda \delta}\right)\|x\|+\varepsilon^{2} \delta$.

Since, the Jacobian matrix $[\partial f / \partial x]$ is uniformly bounded on $D$, let

$$
\left\|\frac{\partial f}{\partial x}(t, x)\right\| \leq L, \quad \forall x \in D .
$$

Then,

$$
\|f(t, x)\| \leq L\|x\|
$$

and $\phi(\tau ; t, x)$ satisfies the lower bound (see Appendix, Lemma 1)

$$
\|\phi(\tau ; t, x)\|^{2} \geq\|x\|^{2} e^{-2 L(\tau-t)} .
$$

Hence, using (23) in (20), $V(t, x)$ satisfies the lower bound

$V(t, x) \geq \int_{t}^{t+\delta} e^{-2 L(\tau-t)} d \tau\|x\|^{2}=\frac{1}{2 L}\left(1-e^{-2 L \delta}\right)\|x\|^{2}$.

Thus, from (21) and (24), $V(t, x)$ satisfies the first inequality of the theorem with

$$
\begin{aligned}
c_{1} & =\frac{1}{2 L}\left(1-e^{-2 L \delta}\right), & c_{2} & =\frac{k^{2}}{2 \lambda}\left(1-e^{-2 \lambda \delta}\right) \\
c_{3} & =\frac{2 \varepsilon k}{\lambda}\left(1-e^{-\lambda \delta}\right), & c_{4} & =\varepsilon^{2} \delta
\end{aligned}
$$

To calculate the derivative of $V(t, x)$ along the system trajectories, the following sensitivity functions are defined

$$
\phi_{t}(\tau ; t, x)=\frac{\partial}{\partial t} \phi(\tau ; t, x) ; \quad \phi_{x}(\tau ; t, x)=\frac{\partial}{\partial x} \phi(\tau ; t, x) .
$$

Then,

$$
\begin{aligned}
\frac{\partial V}{\partial t}+\frac{\partial V}{\partial x} f(t, x)= & \phi^{T}(t+\delta ; t, x) \phi(t+\delta ; t, x) \\
& -\phi^{T}(t ; t, x) \phi(t ; t, x) \\
& +\int_{t}^{t+\delta} 2 \phi^{T}(\tau ; t, x) \phi_{t}(\tau ; t, x) d \tau \\
& +\int_{t}^{t+\delta} 2 \phi^{T}(\tau ; t, x) \phi_{x}(\tau ; t, x) d \tau \\
& \times f(t, x) \\
= & \phi^{T}(t+\delta ; t, x) \phi(t+\delta ; t, x) \\
& +\int_{t}^{t+\delta} 2 \phi^{T}(\tau ; t, x)\left[\phi_{t}(\tau ; t, x)\right. \\
& \left.+\phi_{x}(\tau ; t, x) f(t, x)\right] d \tau-\|x\|^{2} .
\end{aligned}
$$

Using the fact that (see Appendix, Lemma 2)

$$
\phi_{t}(\tau ; t, x)+\phi_{x}(\tau ; t, x) f(t, x) \equiv 0, \quad \forall \tau \geq t
$$

we get

$$
\begin{aligned}
\frac{\partial V}{\partial t}+\frac{\partial V}{\partial x} f(t, x)= & \phi^{T}(t+\delta ; t, x) \phi(t+\delta ; t, x)-\|x\|^{2} \\
\leq & \left(k\|x\| e^{-\lambda \delta}+\varepsilon\right)^{2}-\|x\|^{2} \\
\leq & -\left(1-k^{2} e^{-2 \lambda \delta}\right)\|x\|^{2} \\
& +2 \varepsilon k e^{-\lambda \delta}\|x\|+\varepsilon^{2} .
\end{aligned}
$$

Therefore, the second inequality of the theorem is satisfied with

$$
c_{5}=1-k^{2} e^{-2 \lambda \delta}, \quad c_{6}=2 \varepsilon k e^{-\lambda \delta}, \quad c_{7}=\varepsilon^{2} .
$$

\section{PARAmEter ESTIMATION AND TRACKING}

Theorem 3: Suppose there exists a parameter estimator such that the parameter estimation error $\tilde{\theta}(t)$ satisfies

$$
\|\tilde{\theta}(t)\| \leq k\|\tilde{\theta}(0)\| e^{-\lambda t}+\varepsilon
$$

then, the control law (9) in conjunction with the parameter estimator yields globally uniformly ultimately bounded (GUUB) tracking stability for the system in (1) and all closed-loop signals are bounded.

Proof: Theorem 2 implies that there exists a function $V_{c l f}(t, \tilde{\theta})$ such that

$$
c_{1}\|\tilde{\theta}\|^{2} \leq V_{c l f} \leq c_{2}\|\tilde{\theta}\|^{2}+c_{3}\|\tilde{\theta}\|+c_{4}
$$

and

$$
\dot{V}_{c l f} \leq-c_{5}\|\tilde{\theta}\|^{2}+c_{6}\|\tilde{\theta}\|+c_{7}
$$

Define

$$
V_{s}=V_{i s s}+V_{c l f}
$$

where $V_{i s s}$ was defined in (11), which satisfies the following lower and upper bounds:

$$
\begin{gathered}
V_{s} \geq \frac{1}{2} m_{1}\left\|e_{2}\right\|^{2}+c_{1}\|\tilde{\theta}\|^{2} \\
V_{s} \leq \frac{1}{2} m_{2}\left\|e_{2}\right\|^{2}+c_{2}\|\tilde{\theta}\|^{2}+c_{3}\|\tilde{\theta}\|+c_{4} .
\end{gathered}
$$

Taking the time derivative of (31), and using the results of Theorems 1 and 2

$$
\dot{V}_{s} \leq-k_{1}\left\|e_{2}\right\|^{2}+\frac{1}{4 k_{n}}\|\tilde{\theta}\|^{2}-c_{5}\|\tilde{\theta}\|^{2}+c_{6}\|\tilde{\theta}\|+c_{7} .
$$

Using the upper bound of (33) in (34)

$$
\begin{aligned}
\dot{V}_{s} \leq & -2 \lambda_{i s s} V_{s}-\left(c_{5}-2 \lambda_{i s s} c_{2}-\frac{1}{4 k_{n}}\right)\|\tilde{\theta}\|^{2} \\
& +\left(2 \lambda_{i s s} c_{3}+c_{6}\right)\|\tilde{\theta}\|+2 \lambda_{i s s} c_{4}+c_{7}
\end{aligned}
$$

Let $\gamma_{1}=c_{5}-2 \lambda_{i s s} c_{2}-\frac{1}{4 k_{n}} ; \gamma_{2}=2 \lambda_{i s s} c_{3}+c_{6} ; \gamma_{3}=$ $2 \lambda_{i s s} c_{4}+c_{7}$, where $\gamma_{1}, \gamma_{2}, \gamma_{3}$ are positive constants such that the following sufficient conditions should be satisfied

$$
k_{n}>\frac{1}{4\left(c_{5}-2 \lambda_{i s s} c_{2}\right)}, \quad \lambda_{i s s}<\frac{c_{5}}{2 c_{2}} .
$$


Completing the squares for the second and the third terms in (35)

$$
\dot{V}_{s} \leq-2 \lambda_{i s s} V_{s}+\gamma
$$

where the positive constant $\gamma$ is defined as

$$
\gamma=\frac{\gamma_{2}^{2}}{4 \gamma_{1}}+\gamma_{3}
$$

After solving the differential inequality of (37), we get

$$
V_{s}(t) \leq V_{s}(0) e^{-2 \lambda_{i s s} t}+\frac{\gamma}{2 \lambda_{i s s}}\left(1-e^{-2 \lambda_{i s s} t}\right) .
$$

From (32), we have

$$
\frac{1}{2} m_{1}\left\|e_{2}\right\|^{2} \leq \frac{1}{2} m_{1}\left\|e_{2}\right\|^{2}+c_{1}\|\tilde{\theta}\|^{2} \leq V_{s} .
$$

Then, the following bound on the filtered tracking error $e_{2}(t)$ can be obtained:

$$
\left\|e_{2}(t)\right\|^{2} \leq \frac{2 V_{s}(0)}{m_{1}} e^{-2 \lambda_{i s s} t}+\frac{\gamma}{m_{1} \lambda_{i s s}} .
$$

Therefore,

$$
\left\|e_{2}(t)\right\| \leq \sqrt{\frac{2 V_{s}(0)}{m_{1}}} e^{-\lambda_{i s s} t}+\sqrt{\frac{\gamma}{m_{1} \lambda_{i s s}}} .
$$

Using (5), a similar bound for $\left\|e_{1}(t)\right\|$ can be derived, and hence, $q(t), \dot{q}(t)$ are bounded and the control torque (9) is bounded.

Remark 1: Substituting for $c_{2}$ and $c_{5}$ from (25) and (27) in to the gain condition for $\lambda_{i s s}$ in (36), we have

$$
\lambda_{i s s}<\lambda \frac{\left(1-k^{2} e^{-2 \lambda \delta}\right)}{k^{2}\left(1-e^{-2 \lambda \delta}\right)} .
$$

The expression on the RHS is of the order of $\mathcal{O}(\lambda)$. Thus, the condition in (42) indicates that the decay rate of the tracking error is less than that of the estimator. In other words, the parameter estimator should have a fast convergence in order to ensure a good tracking performance. Similarly, from (40) using (38), we have

$$
\begin{aligned}
\frac{\gamma}{m_{1} \lambda_{i s s}}= & \frac{1}{m_{1} \lambda_{i s s}}\left(\frac{\gamma_{2}^{2}}{4 \gamma_{1}}+\gamma_{3}\right) \\
= & \frac{1}{m_{1} \lambda_{i s s}}\left(\frac{\left(2 \lambda_{i s s} c_{3}+c_{6}\right)^{2}}{4\left(c_{5}-2 \lambda_{i s s} c_{2}-1 / 4 k_{n}\right)}\right) \\
& +\frac{1}{m_{1} \lambda_{\text {iss }}}\left(2 \lambda_{i s s} c_{4}+c_{7}\right) .
\end{aligned}
$$

Substituting $c_{1}, c_{2}, \ldots, c_{7}$ from (25) and (27),

$$
\begin{aligned}
\frac{\gamma}{m_{1} \lambda_{i s s}}= & \frac{4 \varepsilon^{2} k^{2}\left(2 \lambda_{i s s}\left(1-e^{-\lambda \delta}\right)+\lambda e^{-\lambda \delta}\right)^{2}}{\lambda\left(\lambda-\lambda k^{2} e^{-2 \lambda \delta}-\lambda_{i s s} k^{2}\left(1-e^{-2 \lambda \delta}\right)-\frac{\lambda}{4 k_{n}}\right)} \\
& \times \frac{1}{m_{1} \lambda_{i s s}}+\frac{\varepsilon^{2}}{m_{1} \lambda_{i s s}}\left(2 \lambda_{i s s} \delta+1\right)
\end{aligned}
$$

which is of the order of $\mathcal{O}\left(\varepsilon^{2}\right)$, and therefore, from (41), the ultimate bound on $\left\|e_{2}(t)\right\|$ is $\mathcal{O}(\varepsilon)$.

Remark 2: If the estimation error $\tilde{\theta}(t)$ in (28) exponentially converges to zero (i.e., $\varepsilon=0$ ), then from (43), the tracking error also exponentially converges to a zero steady-state.

\section{Simulation STUdY}

In order to validate the theoretical development, a computer simulation was performed on a space robot system. Control of space robots is important for various space applications such as material transport, cooperative autonomous construction, maintenance of space stations and satellites, etc. Specifically, we consider a space robot consisting of a base (mass $m_{0}$ ), and a two-DOF revolute manipulator with equal link lengths $l$ and point masses $m_{1}$ and $m_{2}$ at the end of each link. Although the proposed method can control the full system, for simplicity the proposed adaptive controller is applied only for the motion control of the arm. It is assumed that the attitude of the base can be effectively controlled by other control methods. Neglecting the gravity term in (1), the system dynamics can be derived in a manner similar to [22] so that $M=p_{1} R_{1}+p_{2} R_{2}+p_{3} R_{3}$, where $p_{1}=\frac{m_{0} m_{1}}{m_{c}}, p_{2}=$ $\frac{m_{1} m_{2}}{m_{c}}, p_{3}=\frac{m_{0} m_{2}}{m_{c}}$, where $m_{c}=m_{0}+m_{1}+m_{2}$, and

$$
\begin{gathered}
R_{1}=\left[\begin{array}{ll}
l^{2} & 0 \\
0 & 0
\end{array}\right], R_{2}=\left[\begin{array}{ll}
l^{2} & l^{2} \\
l^{2} & l^{2}
\end{array}\right] \\
R_{3}=\left[\begin{array}{cc}
2\left(1+c_{2}\right) l^{2} & \left(1+c_{2}\right) l^{2} \\
\left(1+c_{2}\right) l^{2} & l^{2}
\end{array}\right]
\end{gathered}
$$

where $c_{2}=\cos \left(q_{2}\right) \cdot V_{m}=p_{3} R_{4}$, where

$$
R_{4}=\left[\begin{array}{cc}
-2 l^{2} s_{2} \dot{q}_{2} & -l^{2} s_{2} \dot{q}_{2} \\
l^{2} s_{2} \dot{q}_{1} & 0
\end{array}\right], \text { where } s_{2}=\sin \left(q_{2}\right) .
$$

A continuous friction model similar to [23] is used, $F=\gamma_{1} R_{5}+\gamma_{2} R_{6}+\gamma_{3} \dot{q}$, where $R_{5}=\tanh (0.1 \dot{q})-$ $\tanh (0.2 \dot{q}), R_{6}=\tanh (0.15 \dot{q})$. It is assumed that the link length $l$ is known, while the masses $m_{0}, m_{1}, m_{2}$ (equivalently, $p_{1}, p_{2}, p_{3}$ ) and the friction coefficients $\gamma_{1}, \gamma_{2}, \gamma_{3}$ are unknown. Defining $\zeta_{1}=\ddot{q}_{d}+\alpha_{1} \dot{e}_{1}, \zeta_{2}=$ $\dot{q}_{d}+\alpha_{1} e_{1}$, and comparing with (8), we have $Y=$ $\left[\begin{array}{llllll}R_{1} \zeta_{1} & R_{2} \zeta_{2} & \left(R_{3} \zeta_{1}+R_{4} \zeta_{2}\right) & R_{5} & R_{6} & \dot{q}\end{array}\right]$ and the unknown parameter $\theta=\left[\begin{array}{llllll}p_{1} & p_{2} & p_{3} & \gamma_{1} & \gamma_{2} & \gamma_{3}\end{array}\right]^{T}$. An arbitrary parameter estimator is constructed such that the bound in (28) is satisfied. The parameter estimates are initialized to half of their true values and the resulting parameter estimation error is shown in Fig. 1. The adaptive control law in (9) with the control gains chosen by trial and error is employed to track a desired sinusoidal trajectory. The position tracking error converges to a small bound (shown in Fig. 2) as proved by the stability analysis.

Fig. 1. $\tilde{\theta}(t)$ exponentially converges to a small bound.

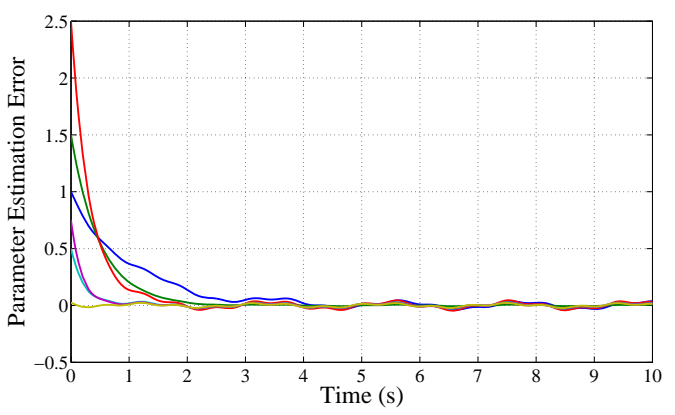


Fig. 2. Position tracking error.

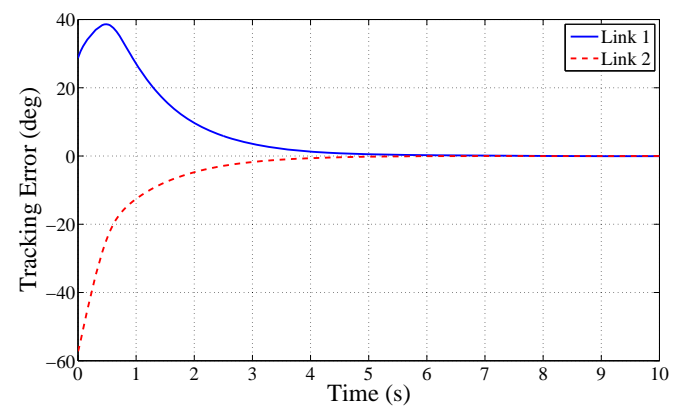

\section{CONCLUSION AND FUtURE WORK}

Using a converse Lyapunov-like theorem, sufficient gain conditions were derived under which any exponential parameter estimator can be used with an adaptive controller to achieve tracking with a performance bound on the tracking error. The adaptive controller is augmented with a nonlinear damping term which makes the closed-loop system input-tostate stable enabling controller/update law separation. The current development is based on Euler-Lagrange dynamic systems. Future work will focus on extending the result to more general systems with parametric uncertainty.

\section{REFERENCES}

[1] J. J. Slotine and W. Li, Applied Nonlinear Control. Upper Saddle River: Prentice-Hall, 1991.

[2] — - "Composite adaptive control of robot manipulators," Automatica, vol. 25, no. 4, pp. 509-519, Jul. 1989.

[3] R. L. Leal and C. C. DeWit, "Passivity based adaptive control for mechanical manipulators using LS-type estimation," IEEE Trans. Automat. Contr., vol. 35, no. 12, pp. 1363-1365, Dec. 1990.

[4] V. H. Nascimento and A. H. Sayed, "Lp-robustness and exponential convergence of some estimation schemes in adaptive control," in Proc. Amer. Control Conf., vol. 1, 1997, pp. 338-342.

[5] N. Sadegh and R. Horowitz, "An exponentially stable adaptive control law for robot manipulators," IEEE Trans. Robot. Automat., vol. 6, no. 4, pp. 491-496, Aug. 1990.

[6] Y. Tang and M. A. Arteaga, "Adaptive control of robot manipulators based on passivity," IEEE Trans. Automat. Contr., vol. 39, no. 9, pp. 1871-1875, Sept. 1994.

[7] W. E. Dixon, M. S. de Queiroz, D. M. Dawson, and T. J. Flynn, "Adaptive tracking and regulation of a wheeled mobile robot with controller/update law modularity," IEEE Trans. Contr. Syst. Technol., vol. 12, no. 1, pp. 138-147, Jan. 2004.

[8] M. Krstic and P. V. Kokotovic, "Adaptive nonlinear design with controller-identifier separation and swapping," IEEE Trans. Automat. Contr., vol. 40, no. 3, pp. 426-440, Mar. 1995.

[9] R. H. Middleton and C. G. Goodwin, "Adaptive computed torque control for rigid link manipulators," System and Control Letters, vol. 10, pp. 9-16, 1988.

[10] M. S. de Queiroz, D. M. Dawson, and M. Agarwal, "Adaptive control of robot manipulators with controller/update law modularity," Automatica, vol. 35, pp. 1379-1390, 1999.

[11] M. Krstic, I. Kanellakopoulos, and P. Kokotovic, Nonlinear and Adaptive Control Design. New York: Wiley, 1995.

[12] R. H. Middleton, "Hybrid adaptive control of robot manipulators," in Proc. IEEE Conf. on Decision and Control, Austin, TX, 1988, pp. 1592-1597.

[13] S. Boyd and S. Sastry, "On parameter convergence in adaptive control," System and Control Letters, vol. 3, no. 6, pp. 311-319, Dec 1983.

[14] R. M. Johnstone, C. R. Johnson, R. R. Bitmead, and B. D. O. Anderson, "Exponential convergence of recursive least squares with exponential forgetting factor," in IEEE Conf. on Decision and Control, vol. 21, 1982, pp. 994-997.
[15] P. V. Kokotovic, "The joy of feedback: nonlinear and adaptive," IEEE Control Syst. Mag., vol. 12, no. 3, pp. 7-17, Jun. 1992.

[16] P. M. Patre, W. MacKunis, K. Dupree, and W. E. Dixon, "Modular adaptive control of uncertain Euler-Lagrange systems with additive disturbances," IEEE Trans. Automat. Contr, vol. 56, no. 1, pp. 155160, 2011.

[17] P. M. Patre, W. MacKunis, C. Makkar, and W. E. Dixon, "Asymptotic tracking for systems with structured and unstructured uncertainties," IEEE Trans. Contr. Syst. Technol., vol. 16, no. 2, pp. 373-379, 2008.

[18] F. L. Lewis, C. Abdallah, and D. Dawson, Control of Robot Manipulators. New York: MacMillan Publishing Co., 1993.

[19] A. G. Kelkar and S. Joshi, Control of Nonlinear Multibody Flexible Space Structures, ser. Lecture Notes in Control and Information Sciences. Springer-Verlag, 1996.

[20] R. Ortega and M. Spong, "Adaptive motion control of rigid robots: a tutorial," in Proc. IEEE Conf. on Decision and Control, 1988, pp. $1575-1584$.

[21] H. K. Khalil, Nonlinear Systems, 3rd ed. New Jersey: Prentice-Hall, Inc., 2002.

[22] Y. Xu, H. Shum, T. Kanade, and J. Lee, "Parameterization and adaptive control of space robot systems," IEEE Trans. Aerosp. Electron. Syst., vol. 30, no. 2, pp. 435-451, 1994.

[23] C. Makkar, W. E. Dixon, W. G. Sawyer, and G. Hu, "A new continuously differentiable friction model for control systems design," in Proc. IEEE/ASME Intl. Conf. on Advanced Intelligent Mechatronics, Monterey, CA, 2005, pp. 600-605.

\section{APPENDIX}

Lemma 1: In (23), $\|\phi(\tau ; t, x)\|^{2} \geq\|x\|^{2} e^{-2 L(\tau-t)}$. Proof: From (19) and (22)

$$
\begin{aligned}
\left|\frac{d}{d t}\left[x^{T}(t) x(t)\right]\right| & =\left|2 x^{T}(t) \dot{x}(t)\right| \\
& =\left|2 x^{T}(t) f(t, x)\right| \leq 2 L\|x\|^{2} .
\end{aligned}
$$

Therefore,

$$
-2 L\|x\|^{2} \leq \frac{d}{d t}\left[x^{T}(t) x(t)\right] \leq 2 L\|x\|^{2} .
$$

Solving the differential inequality in (44) such that $\phi(\tau ; t, x)$ denotes the solution of the system starting at $(t, x)$ (i.e., $\phi(t ; t, x)=x)$

$$
\|x\| e^{-L(\tau-t)} \leq\|\phi(\tau ; t, x)\| \leq\|x\| e^{L(\tau-t)} .
$$

Hence, (23) holds.

Lemma 2: In (26), $\phi_{t}(\tau ; t, x)+\phi_{x}(\tau ; t, x) f(t, x) \equiv$ $0, \quad \forall \tau \geq t$.

Proof: The solution $\phi(\tau ; t, x)$ starting at $(t, x)$ can be expressed as

$$
\begin{aligned}
\phi(\tau ; t, x) & =\phi(t ; t, x)+\int_{t}^{\tau} f(s, \phi(s)) d s \\
& =x+\int_{t}^{\tau} f(s, \phi(s)) d s .
\end{aligned}
$$

Therefore,

$$
\phi_{t}(\tau ; t, x)=\frac{\partial x}{\partial t}-f(t, \phi(t))=-f(t, x)
$$

and

$$
\phi_{x}(\tau ; t, x)=\frac{\partial x}{\partial x}=1 .
$$

From (45) and (46), it can be verified that (26) holds. 